\title{
The spin-spin interaction mechanism between quarks and its impact on the mass spectrum of mesons
}

\author{
Engel Roza \\ Philips Research Labs, Eindhoven, The Netherlands (retired) \\ Email: engel.roza@onsbrabantnet.nl
}

\begin{abstract}
A structure-based view on mesons is given, based upon the concept of an archetype quark, described as a pointlike source producing an energy flux, the spatial description of which is derived from Dirac's second dipole moment. This enables to conceive the archetype meson (pion) as a structure that behaves as a one-body anharmonic quantum mechanical oscillator. All mesons appear being excitations of the archetype, thereby allowing a calculation of the mass spectrum without the use of empirical parameters for the masses of the quark flavors. This includes a physically comprehensible analysis of the spin-spin interaction between quarks. It also provides a solution for the eta-etaprime puzzle. Next to this, it is shown that quite some particles that are presently regarded as elementary, have a common root and can be traced back to a few archetypes only.
\end{abstract}

Keywords: quarks; spin-spin interaction; hadronization limit; topquark; eta-etaprime puzzle; mass spectrum

\section{Introduction}

Present-day particle physics theory still struggles with the mass issue, albeit that ever since the discovery of the $125 \mathrm{GeV}$ boson the origin of mass is believed to be understood from the Higgs mechanism. But, whereas defined invariant charge values are attributed to quarks, the mass values of quarks are not very well established, in spite of the fact that the mass values of hadrons can be measured with high precision. The mass relationships between the hadrons still mainly rely upon the phenomenological rules on the masses of constituent quarks, expressed by the Gell-Mann-Okubo formula, which dates back from 1961-1962, [1,2]. In 1980, Zakharov has formulated an empirical set of mass rules, in which the difference between the sum of the masses of the constituent quarks with the hadron mass is attributed to the influence of the spin-spin interaction between quarks [3].The model of this interaction is inherited from an analogy with the interaction of the spin of the orbiting electron with the nuclear spin of the proton in the Hydrogen atom. The strength of this interaction, however, is empirically established. Ever since the work by Shifman, Vainshtein and Zakharov, as published in 1979 [4], the physical mechanism of the spin-spin interaction is attributed to gluons as formulated in a set of QCD rules, now known as the SVZ rules. Present studies in lattice QCD [5] aim to build out this approach to give further theoretical support.

In this article, I wish to show that the interpretation of quarks as Dirac particles as described in [6] allows a more simple description of the spin-spin interaction that nicely fits to the empirical observations as made in Zakharov's paper in 1980, which still to-day are invoked in tutorials to give some clue for understanding the concept of constituent quarks and their influence on the mass spectrum of hadrons [7, p.184]. The particular interpretation of the quark that we shall discuss is based upon the awareness of a second anomalous dipole moment next to the well-known anomalous angular dipole moment of a Dirac particle. Whereas Dirac gave a magnetic dipole interpretation to the latter one, he could not justify the first one as an electric dipole moment. This, in spite of his own derivation, Because in the case of electrons the second dipole moment escapes from observation, this second dipole moment has been further ignored. In [6[, updated in [26], I have given reasons why this ignorance is not justified for other Dirac particles but electrons. 
It is my aim in this article to show that the existence of this second anomalous dipole moment allows a very similar description of the spin-spin interaction mechanism between quarks as in the Maxwellian type spin-spin interaction in the description of the hyperfine spectrum of the Hydrogen atom. This will give a comprehensible view on the mass spectrum of hadrons straight from theory, without empirics or heuristics. It will bring some additional merits, such as the explanation for the big gap between the masses of the constituent $u / d, s, c$ and $b$ quarks and that of the topquark and an explanation for the absence of mesons beyond the bottomed ones (the hadronization limit). It will also give a solution for the well-known " $\eta-\eta^{\prime}$ puzzle", by explaining why the $\eta$ meson and the $\eta^{\prime}$ meson don't obey Zakharov's mass rules [7, p.172]. Furthermore, the relationship will be discussed between the masses of the W/Z bosons, the topquark and the Higgs boson. It will be shown that these relationships allow to reduce the number of elementary particles quite substantially.

The focus of the analysis in this article is on mesons, but the results apply equally well to baryons. The work is an extension and improvement of earlier studies by the author on the mass spectrum of hadrons. In section 2, the structure of mesons will be described in terms of a potential field for quarks derived from Dirac's Hamiltonian. In the third section the classification of mesons will be rediscussed. Primarily for showing the mismatch of the $\eta$ meson and the $\eta^{\prime}$ meson with the commonly adopted SU(3) classification scheme. In section 4, the mass model of mesons will be further discussed. These sections serve as an introduction for the analysis of spin-spin interaction mechanism between quarks to be described in section 5 . In section 6 , an explanation will be given for the hadronization limit in the mass spectrum of mesons. In section 7, the relationship between the nuclear W/Z bosons with the topquark and the $125 \mathrm{GeV}$ Higgs boson will be discussed. In the final sections 8 and 9 , the results of the study will be summarized and discussed.

The equations in this article will be formulated in scientific notation and the quantities will be expressed in SI units.

\section{The structure of mesons and their classification}

The mass spectrum of mesons can be calculated from a structural model of the pion. This model will be based upon the assignment of a scalar field $\Phi(r)$ to a quark that consists of a far field component $\Phi_{F}(r)$ and a near field component $\Phi_{N}(r)$, such that,

$\Phi(r)=\Phi_{F}(r)+\Phi_{N}(r)$

The far field component is the classical potential field shielded by a background field, i.e.,

$\Phi_{F}(r)=-\Phi_{0} \frac{\exp (-\lambda r)}{\lambda r}$

In which $\Phi_{0}$ is a strength parameter and in which $\lambda$ is a parameter for spatial normalization. The near field is the field of Dirac's linear dipole moment $[8,6]$. As a consequence, the quark's potential field along the axis set up between the poles of the linear dipole has the format,

$\Phi(x)=\Phi_{0} \exp (-\lambda x)\left(\frac{1}{(\lambda x)^{2}}-w \frac{1}{\lambda x}\right)$,

In which $w$ is a dimensionless weighing factor that relates the strength of the far field to the near field. This model and its justification have been extensively described in [9]. Conceiving the pion as a 
structure in which a quark couples to the field of the antiquark with a generic quantum mechanical coupling factor $g$, the pion can be modeled as the one-body equivalent of a two-body oscillator, described by the equation for its wave function $\psi$,

$$
-\frac{\hbar^{2}}{2 m_{m}} \frac{\mathrm{d}^{2} \psi}{\mathrm{dx}^{2}}+\{U(d+x)+U(d-x)\} \psi=E \psi ; \quad U(x)=g \Phi(x),
$$

In which $\hbar$ is Planck's reduced constant, $2 d$ the quark spacing, $m_{m}$ is the effective mass of the center, $V(x)=U(d+x)+U(d-x)$ its potential energy, and $E$ the generic energy constant, which is subject to quantization. It will be clear from (1-4) that the potential energy $V(x)$ can be expanded as,

$$
V(x)=U(d+x)+U(d-x)=g \Phi_{0}\left(k_{0}+k_{2} \lambda^{2} x^{2}+\ldots .\right),
$$

In which $k_{0}$ and $k_{2}$ are dimensionless coefficients that depend on the spacing $2 d$ between the quarks.

In spite of the resemblance with a classical quantum mechanical oscillator, the model is relativistic, because the mass in the wave equation does not represent the individual masses of the two bodies. Instead, it is an equivalent mass that captures the energy of the field. Furthermore, it has to be kept in mind that this model holds in the center of mass frame. Hence, a lab frame interpretation will need a relativistic correction. To facilitate the analysis, (4) is normalized as,

$-\alpha_{0} \frac{\mathrm{d}^{2} \psi}{\mathrm{d} x^{\prime 2}}+V^{\prime}\left(x^{\prime}\right)=E^{\prime} \psi$

in which $\alpha_{0}=\frac{\lambda^{2} \hbar^{2}}{2 m_{m} g \Phi_{0}}, x^{\prime}=x \lambda, d^{\prime}=d \lambda, E^{\prime}=\frac{E}{g \Phi_{0}}, U^{\prime}\left(x^{\prime}\right)=\frac{U(\lambda x)}{g \Phi_{0}}$ and

$V^{\prime}\left(x^{\prime}\right)=U^{\prime}\left(d^{\prime}+x^{\prime}\right)+U^{\prime}\left(d^{\prime}-x^{\prime}\right)=k_{0}+k_{2} x^{\prime 2}+\ldots \ldots$

Moreover, from [9, eq. (24)],

$\alpha_{0}=\frac{k_{0}^{2}}{2 k_{2}}$

Normalized quantities in this text will be indicated by a "prime" (').

Further evaluation is hampered by lack of knowledge of the value of the dimensionless factor $w$. This knowledge can be gained by adopting the Lagrangian description of the nuclear energetic background field, such as hypothesized in the Standard Model of particle physics. Generically, the scalar format of the Lagrangian density in classical field theory is written as

$$
\mathcal{L}=-\frac{1}{2}(\nabla \Phi)^{2}+U(\Phi)+\rho \Phi
$$


in which the source or assembly of sources $\rho\left(x_{1}, x,{ }_{2} x_{3}\right)$ is described by a time-independent function. Usually, knowledge of $U(\Phi)$ allows deriving (by application of the Euler-Lagrange equation) a wave function $\Phi(r)$ for a pointlike particle that creates the field. The nuclear field in particle physics is known as the Higgs field, with the axiomatic format [7, p. 365],

$U(\Phi)=-\frac{1}{2} \mu_{c}^{2} \Phi^{2}+\frac{1}{4} \lambda_{c}^{2} \Phi^{4}$

In which $\mu_{c}$ and $\lambda_{c}$ are two characteristic parameters. Unfortunately the particular format of this (broken) field prevents deriving an analytical solution $\Phi(r)$ of (8) subject to (9). However, a numerical procedure allows deriving a two-parameter expression for $\Phi(r)$ that closely approximates a true analytical solution. The result is [9],

$$
\Phi(r)=\Phi_{0} \frac{\exp [-\lambda r]}{\lambda r}\left(\frac{\exp [-\lambda r]}{\lambda r}-1\right) \text { with } \frac{1}{2} \mu_{c}^{2}=1.06 \lambda^{2} \text { and } \frac{1}{4} \lambda_{c}^{2}=32.3 \frac{\lambda^{2}}{\Phi_{0}^{2}}
$$

This result can be written as the sum of a far field and a near field, such that,

$$
\Phi(r)=\Phi_{F}(r)+\Phi_{N}(r) \text { with } \Phi_{F}(r)=-\Phi_{0} \frac{\exp (-\lambda r)}{\lambda r} \text { and } \Phi_{N}(r)=\Phi_{0} \frac{\exp (-2 \lambda r)}{(\lambda r)^{2}}
$$

Unfortunately, this radial symmetric solution is not viable, because the near field violates the renormalization constraint. The format (11), however, resembles the viable unidirectional format (3) that doesn't suffer from this problem. Fitting the two expressions along the $x$-axis allows deriving a quantitative value for $w$. A very precise fit is obtained for $w=1 / 0.555$. This precise fit allows to adopt the two-parameter solution

$\Phi(x)=\Phi_{F}(x)+\Phi_{N}(x)$ with $\Phi_{F}(\mathrm{x})=-\Phi_{0} \frac{\exp (-\lambda x)}{\lambda x}$ and $\Phi_{N}(\mathrm{x})=\Phi_{0} \frac{\exp (-2 \lambda x)}{(\lambda x)^{2}}$

for further elaboration. The coefficients $k_{0}\left(d^{\prime}\right)$ and $k_{2}\left(d^{\prime}\right)$ can be straightforwardly calculated from (6) and (12) as,

$$
\begin{aligned}
& k_{0}=2\left(\frac{\exp \left(-2 d^{\prime}\right)}{d^{\prime 2}}-\frac{\exp \left(-d^{\prime}\right)}{d^{\prime}}\right) \\
& k_{2}=\frac{\exp \left(-2 d^{\prime}\right)}{d^{\prime 4}}\left(6+4 d^{\prime 2}+8 d^{\prime}\right)-\frac{\exp \left(-d^{\prime}\right)}{d^{\prime 2}}\left(2+d^{\prime}+\frac{2}{d^{\prime}}\right)
\end{aligned}
$$

The two quarks in the meson settle in a state of minimum energy, at a spacing $2 \lambda d=2 d_{\min }^{\prime}$, such that [9],

$d_{\text {min }}^{\prime}=\lambda d=0.853 ; k_{0}=-1 / 2$ and $k_{2}=2.36$

At this point I would like to emphasize here that the theory as will be developed in this article does not depend on the acceptance of the symmetric symmetry breaking (SSB), known as the "Higgs mechanism" [10]. As already mentioned, the reason for bringing it up is to gain some knowledge 
about the magnitude of the factor $w$ in the basic relationship (3). Not more, not less. The additional merit is that consistency is maintained with previous work [9].

As long as $2 \lambda d=2 d_{\min }^{\prime}$ is kept unchanged, $k_{2}, k_{0}$ and $\alpha_{0}$ are constant. The values $k_{0}$ and $k_{2}$ are not adjustable, they are established by theory. Together with the adjustables $\Phi_{0}, \lambda$, spin and isospin (see later in this article) they determine all properties of the hadrons.

The graphs shown in figure 1 illustrate the relationship between the quark's field $\Phi(x)$ and the functional format of its potential energy $U(\Phi)$ derived from it [9]. This characteristic implies that any quark is repelled by any other quark under influence of the far field, but attracted by the near field, thereby giving rise to mesons as stable two-quark junctions and baryons as three-quark junctions.
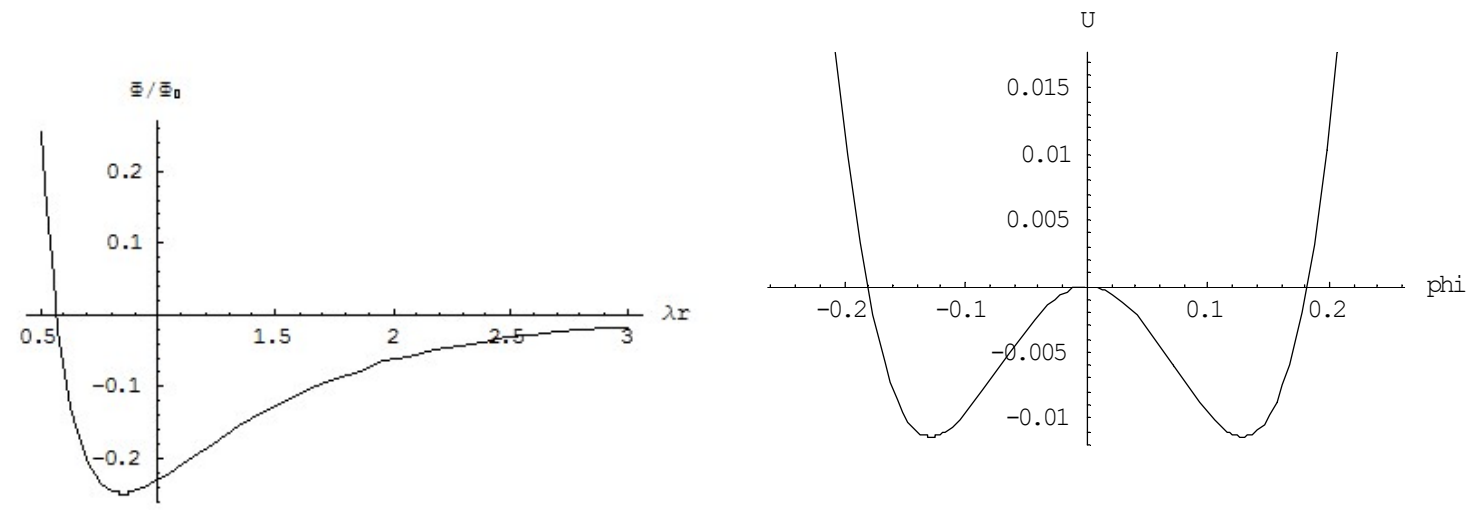

Figure 1. (Left) The quark's scalar field $\Phi / \Phi_{0}$ as a function of the normalized radius $\lambda x$; (Right) The Higgs field $U(\Phi)$ retrieved from the spatial expression.

The archetype, the pion, is the two-quark oscillator in its ground state. The first excitation state transforms a pion into a kaon. The mass ratio between the two is the same as the mass ratio of the normalized energy constants $E^{\prime}-k_{0}$. This is not trivial and it reflects the basic theorem of the theory. This theorem states that the energy wells of the two quarks are not massive. Instead, the mass attribute of two-quark junctions (mesons) and three-quark junctions (baryons) is made up by the vibration energy as expressed by the energy state of the quantum mechanical oscillator that they build. The distribution of this mass over constituent quarks is a consequence of this mechanism. Unfortunately, the analytical calculation of the $E^{\prime}-k_{0}$ ratio of kaons over pions, is only possible for the quadratic approximation of the series expansion of the potential energy $V^{\prime}\left(z^{\prime}\right)$. A more accurate calculation requires a numerical approach. A procedure to do so has been documented in [11, Appendix C]. It shows that some simple lines of code in Wolfram's Mathematica [12] may do the job. The numerically calculated ratio of the energy constants appears to be 3.57 instead of 3 as it would have been in the harmonic case. The result explains the excitation of the $137 \mathrm{MeV} / \mathrm{c}^{2}$ pion mass to the $490 \mathrm{MeV} / \mathrm{c}^{2}$ mass of the pseudoscalar kaon. This result gives a substantial support for the viability of the theory as will be further developed in this article. This result also gives rise to the question if other mesons can be regarded as a result from enhanced excitation. Table I gives a survey of the calculated ratios for higher excitation ratios. It gives the pseudoscalar $\eta^{\prime}$ meson as a candidate from second level excitation. The table gives no candidate for third level excitation. Later in this article, l'll show that the corresponding level of energy would imply a meson state with a positive value for the binding energy (as is reflected in the value of $k_{0}$ ), which prevents a sustainable quasi-stable configuration. 
Table I: meson excitations

\begin{tabular}{|l|l|l|l|}
\hline Bottom level & $E_{\text {bind }}^{\prime}=-1 / 2$ & mass ratio & mass in MeV/c ${ }^{2}$ \\
\hline Ground state & $E_{0}^{\prime}-E_{\text {bind }}^{\prime}=0.84$ & 1 & $\begin{array}{l}137 \\
\text { (pion }=135-140)\end{array}$ \\
\hline First excitation & $E_{1}^{\prime}-E_{\text {bind }}^{\prime}=3.00$ & 3.57 & $\begin{array}{l}489 \\
(\text { kaon }=494-498)\end{array}$ \\
\hline Second excitation & $E_{2}^{\prime}-E_{\text {bind }}^{\prime}=6.06$ & 7.21 & $\begin{array}{l}988 \\
\left(\eta^{\prime}=958\right)\end{array}$ \\
\hline Third excitation & $E_{3}^{\prime}-E_{\text {bind }}^{\prime}=9.94$ & 11.83 & $? ? ?$ \\
\hline
\end{tabular}

In this calculation, the electromagnetic interactions have been ignored, because their influence is considered being of second order as compared to the nuclear interaction. Fine tuning is required to establish the differences between charged mesons and neutral mesons. Interestingly, the kaon energy does not only correspond with the energy of a pion in its state of first excitation, but also with the ground state energy of two heavier quarks at smaller spacing. Therefore, the excitation mechanism is potentially subject to bootstrapping. It shows many excitation and de-excitation routes, resulting in a quasi-chaotic, but nevertheless deterministic, mass spectrum of mesons. So, the meson's mass spectrum can be explained from excitations of a basic structure consisting of two identical quarks. The pion-kaon-etaprime sequence is just the hat-stand of a framework for the assignment of constituent quark masses. A major refinement needs the inclusion of the influence of spin-spin interactions between quarks. Before doing so, let us first reconsider the meson's classification scheme.

\section{The classification of mesons}

The classification of hadrons is based upon the spin concept. A Dirac spinor of a pointlike fermion may manifest itself into four states: two for the particle/antiparticle state and two for the state of twist of the components of the spinor. It is common to express the latter in terms of "spin up" and "spin down". This notion allows a symbolic notation for the spinor state of an $a \bar{b}$ meson: $\uparrow \uparrow, \downarrow \downarrow, \uparrow \downarrow, \downarrow \uparrow$, and ( sometimes) $\uparrow \downarrow \pm \downarrow \uparrow$ mixed states. Nuclear spin has got a mechanical interpretation as intrinsic angular momentum. This enables, for instance, to differentiate between pseudoscalar mesons and vector mesons. Pseudoscalar mesons have their "spins antiparallel" and vector mesons have their "spins parallel".

Next to the nuclear spin there is another attribute with similar characteristics, known as isospin. Isospin has been introduced in quantum physics to explain things that cannot be explained otherwise. Originally, for the purpose to distinguish a neutron from a proton. Eventually, to distinguish an $u$ quark from a $d$ quark. Isospin behaves similarly as nuclear spin. It looks as if Dirac's equation hides a second dipole moment next to the angular one. We now know that this is the case indeed $[6,26]$. This observation implies that the status of a quark is a threefold one. Next to the particle/antiparticle state and the nuclear spin state associated with the elementary angular momenta $\hbar$, there is an additional state associated with a dipole moment $\hbar / c$, which can be conveniently denoted as an isospin state. This allows conceiving the meson as a structure shown in figure 2. Although the individual isospin dipole moments may point into two different directions, their mutual orientation should be opposite for guaranteeing a stable structure. Taking this into consideration, we may assemble Table II in which the possible configurations of the archetype meson 
are shown. The $\uparrow$ symbol is for upward isospin. This means that the horizontal dipole moment of the quark is in inwardly directed. The $\downarrow$ symbol is for the outward direction. It would be misleading to identify these symbols in terms of the common coding $u$ and $d$. This is due to the difference in position of the center-of-mass observer and the lab frame observer. Whereas inwardly directed particles are in the same state for the center-of-frame observer, they are in a different state for the lab frame observer. The table shows the center-of-mass symbolic coding next to the conventional $u / d$ coding. The upper bar in $\bar{u}$ denotes that the $u$ quark is in antiparticle state. The up-state nuclear spin of an $u$ quark is denoted as $u$, its down-state is denoted by lower bar in $\underline{u}$. Mutatis mutandis for $d$ quarks. Note that this coding scheme reveals the difference between the $\rho^{0}$ meson and the $\omega$ meson.

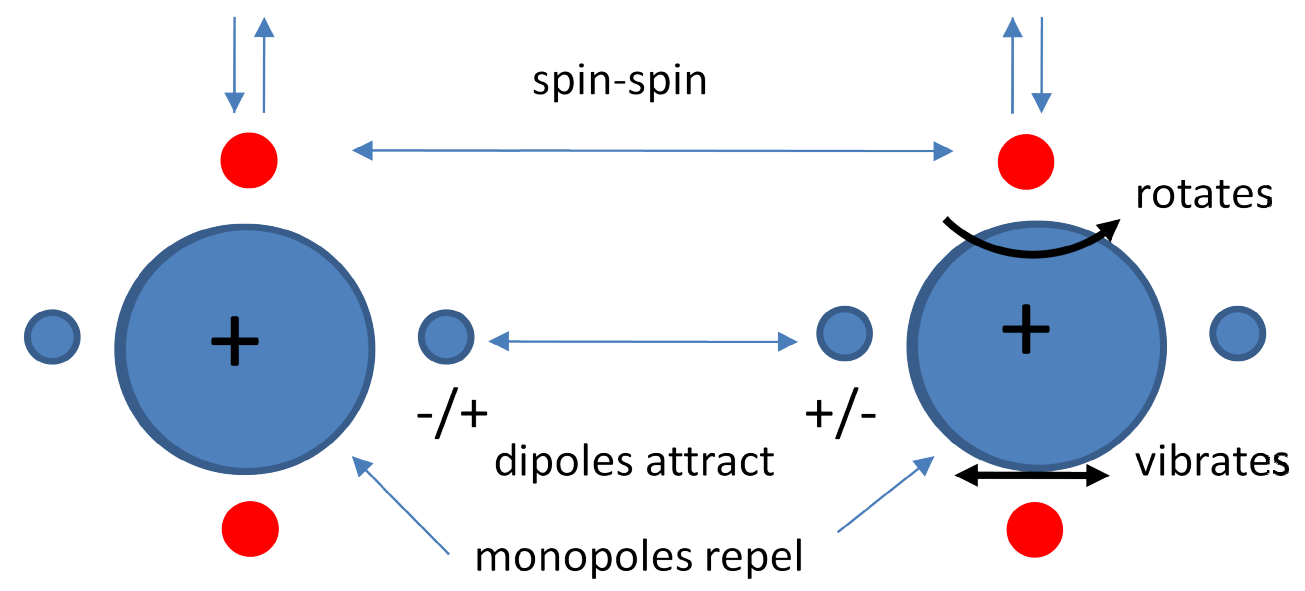

Fig. 2. A quark has two real dipole moments, hence two dipoles. One of these (horizontally visualized) is polarisable in a scalar potential field. The other one (vertically visualized) is not. The dipole moments are subject to spin statistics. However, the polarity of the horizontal one is restrained by the bond: the horizontal dipoles are only oriented in the same direction: either inward to the centre or outward from the centre.

Table II: archetype mesons

\begin{tabular}{|l|l|l|l|l|l|l|l|l|}
\hline \multirow{3}{*}{ meson } & $\begin{array}{c}\text { equivalent } \\
\text { symbolic codes }\end{array}$ & $\begin{array}{l}\text { pseudoscalar } \\
\text { coding }\end{array}$ & symb & $\begin{array}{l}\text { spin } \\
\text { sum (Q) }\end{array}$ & $\begin{array}{l}\text { vector mode } \\
\text { coding }\end{array}$ & symb & $\begin{array}{l}\text { isospin } \\
\text { sum (Q) }\end{array}$ \\
\hline \multirow{4}{*}{$q q$} & $\uparrow \uparrow$ & $\uparrow \uparrow$ & $\underline{u} \bar{d}$ & $\pi^{+}$ & 1 & $u \bar{d}$ & $\rho^{+}$ & 1 \\
\cline { 2 - 10 } & $\downarrow \downarrow$ & $\downarrow \downarrow$ & $\underline{d} \bar{u}$ & $\pi^{-}$ & -1 & $d \bar{u}$ & $\rho^{-}$ & -1 \\
\cline { 2 - 10 } & $\uparrow \uparrow+\downarrow \downarrow$ & $\uparrow \downarrow$ & $\underline{u} \bar{d}+\underline{d} \bar{u} / 2$ & $\pi^{0}$ & 0 & $u \bar{d}+d \bar{u} / 2$ & $\rho^{0}$ & 0 \\
\cline { 2 - 10 } & $\uparrow \uparrow-\downarrow \downarrow$ & $\downarrow \uparrow$ & & & & $u \bar{d}+\underline{d} \underline{u} / 2$ & $\omega$ & 0 \\
\hline
\end{tabular}

The electric charge shows up as a holistic property for the structure (as the isospin sum) without a need to assign individual charges to the components. The individual charges are irrelevant.

Table III is an extension by including $s$ quarks and $c$ quarks. At this point, I wish to point out an interesting phenomenon. It has to do with the $u \bar{s}$ and $\bar{u} s$ compositions. Here, a particular feature pops up. This feature is a constraint on the isospin mode of the strange quark $s$. The isospin mode of the $s$-quark is only down, while the isospin mode of the antiquark $\bar{s}$ is only up. Moreover, mixed modes, such as in the case of the $u / d$ mesons, seem to disappear. A more close inspection reveals 
that this is jumping to conclusions without a physical interpretation. As shown in Table II, the updown configurations shown in the third column are a coding convention for a mixed state. In fact, this holds for the mixed-flavor mesons in Table III as well. The reason for the missing uni-polar states of the mixed-flavor mesons has to do with the origin of the heavier flavor quarks. As to be explained later in this article, the mixed-flavor mesons like, for instance, type $u s$, are the result of the intermediate steps in the excitation process of uni-flavor mesons, like for instance, $u u \rightarrow s s$. In that process the (scalar dipole) polarity of the heavier flavor is determined by the polarity prior to excitation. As a result, full flip of both polarities cannot be accomplished. This polarity bound to the preceding excitation mode is also the reason why the two quarks of the (neutral) $s \bar{s}$ state can only assume a single state of mutual handiness, excluding either the pseudoscalar meson state or the vector meson state. Later in this article it will be shown that the pseudoscalar state $s \bar{s}$ is not viable. In the Standard Model, these physical phenomena remain hidden in axioms, in which the $u$ and $d$ quarks are defined as an isospin twin of two different flavor quarks.

Table lii: meson classification

\begin{tabular}{|l|l|l|l|l|l|}
\hline meson & $\begin{array}{l}\text { Isospin } \\
\text { modes }\end{array}$ & recode & $\mathrm{Q}$ & $\begin{array}{l}\text { pseudo } \\
\text { scalar }\end{array}$ & vector \\
\hline \multirow{4}{*}{$u \bar{u}$} & $\uparrow \uparrow$ & $u \bar{d}$ & 1 & $\pi^{+}$ & $\rho^{+}$ \\
\cline { 2 - 6 } & $\downarrow \downarrow$ & $\bar{u} d$ & -1 & $\pi^{-}$ & $\rho^{-}$ \\
\cline { 2 - 6 } & \multirow{4}{*}{$u \downarrow \pm \downarrow \uparrow$} & $(u \bar{u}-\bar{d} d) / \sqrt{2}$ & 0 & $\pi^{0}$ & $\rho^{0}$ \\
\cline { 2 - 6 } & $(u \bar{u}+\bar{d} d) / \sqrt{2}$ & 0 & $\mathrm{x}$ & $\omega$ \\
\hline \multirow{3}{*}{$u \bar{s}$} & $\uparrow \uparrow$ & $u \bar{s}$ & 1 & $\mathrm{~K}^{+}$ & $\mathrm{K}^{*+}$ \\
\cline { 2 - 6 } & $\downarrow \uparrow$ & $d \bar{s}$ & 0 & $\mathrm{~K}^{0}$ & $\mathrm{~K}^{* 0}$ \\
\hline $\bar{u} s$ & $\uparrow \downarrow$ & $\bar{d} s$ & 0 & $\overline{\mathrm{K}}^{0}$ & $\overline{\mathrm{K}}^{* 0}$ \\
\cline { 2 - 6 } & $\downarrow \downarrow$ & $\bar{u} s$ & -1 & $\overline{\mathrm{K}}^{-}$ & $\overline{\mathrm{K}}^{*-}$ \\
\hline$s \bar{s}$ & $\downarrow \uparrow$ & $s \bar{s}$ & 0 & $\mathrm{x}$ & $\varphi^{-}$ \\
\hline $\bar{u} c$ & $\uparrow \uparrow$ & $\bar{d} c$ & 1 & $\mathrm{D}^{+}$ & $\mathrm{D}^{*+}$ \\
\hline \multirow{3}{*}{$u \bar{c}$} & $\downarrow \uparrow$ & $\bar{u} c$ & 0 & $\mathrm{D}^{0}$ & $\mathrm{D}^{* 0}$ \\
\hline & $\uparrow \downarrow$ & $u \bar{c}$ & 0 & $\mathrm{D}^{0}$ & $\mathrm{D}^{* 0}$ \\
\hline$s \bar{c}$ & $\downarrow \downarrow$ & $d \bar{c}$ & -1 & $\mathrm{D}^{-}$ & $\mathrm{D}^{*-}$ \\
\hline $\bar{s} c$ & $\uparrow \uparrow$ & $\bar{s} c$ & -1 & $\mathrm{D}_{\mathrm{s}}^{-}$ & $\mathrm{D}_{\mathrm{s}}^{*-}$ \\
\hline$c \bar{c}$ & $\uparrow \downarrow$ & $c \bar{c}$ & 1 & $\mathrm{D}_{\mathrm{s}}^{+}$ & $\mathrm{D}_{\mathrm{s}}^{*+}$ \\
\hline
\end{tabular}

The meson table as shown is somewhat different from the common one because of the absence of the $\eta$ meson and the $\eta^{\prime}$ meson. Usually, these mesons are supposed to be composed as a mixed state of $u, d$ and $s$ quarks. This is justified from the hypothesis that the ground state $J^{P}=0^{-1}$ and $J^{P}=1^{-1}$ mesons should show up in the SU(3) classification over three quark types. A first problem, though is, that no theoretical basis exists why the mixing angles of the homogeneous $u \bar{u}, d \bar{d}$ and $s \bar{s}$ quarks are different for the pseudoscalar state and the vector state. They are just established by empirics. A second problem (to be shown later in this article) is the failure to assign a theoretically based mass value to $\eta$ and $\eta^{\prime}$. This is the well known " $\eta-\eta^{\prime}$ puzzle" [7,p.172]. Therefore, I wish to restrict the meson classification to $\mathrm{SU}(2)$ only. This implies that the origin of the $\eta$ meson, the $\eta^{\prime}$ 
meson and the $\eta_{c}$ meson needs a different explanation, to be discussed later in this article, showing that their mass values can be derived from theoretical principles.

The lower part of Table III is a straightforward extension of the upper part, under assumption that the direction of the charmed isospin is just the opposite of the strange isospin. Having established this coding scheme, it is not difficult to detect the relationship of isospin with the electric charge attribute of mesons. Assigning symbolic values, $+1 / 2$ and $-1 / 2$, to, respectively, up-arrows and downarrows, and subsequent addition, just provides the correct value of the electric charge in units of the elementary charge. It is an alternative view on the Gell-Mann-Nishijima formula $[13,14]$.

\section{The mass model of mesons}

The modeling of mesons as a quantum mechanical oscillator may seem naively simple and may seem defying achievements of the present knowledge on particle physics. Appearances are deceptive. The modeling is quite different from adopting a classical structure, because of the following three reasons,

The first one is given before: the mass in the oscillator description (1) does not represent the individual masses of the two bodies, but it is an equivalent mass that captures the energy of the field. Nevertheless, in accordance with the common quantum mechanical oscillator models, the mass $m_{m}$ can be expressed as [15],

$$
\frac{m_{m} \omega^{2}}{2}=g \Phi_{0} k_{2} \lambda^{2}
$$

In which, in the case of a quadratic polynomial field as in the truncated expression (6), $\omega$ is related with the vibration energy $E_{n}=(n+1 / 2) \hbar \omega$. This is just trivial. The second reason is a dual interpretation of $\hbar \omega$. This has to do with the consideration that the two bodies in the oscillator are unconventional, in the sense that the oscillator is built up by a particle and an antiparticle. As a result, the spacing $2 d_{\text {min }}$ between the quark and the antiquark is bridged by half the wavelength of a single harmonic energetic standing wave (boson) with phase velocity $c$. Hence,

$$
\lambda=\frac{2\left(\hbar \omega_{W}\right) d_{\min }^{\prime}}{\alpha \pi(\hbar c)}
$$

In which $\hbar \omega_{W}$ is the weak interaction boson $(80.4 \mathrm{GeV})$ and where $\alpha$ is a dimensionless constant with order of magnitude 1 , which eventually has been established as $\alpha=0.69$, [9]. This gives the one interpretation for $\hbar \omega$.

(Note: Later in this article it will be shown that, not surprisingly, the spatial parameter $\lambda$ of the background field is related with the Higgs boson, such that $m_{H}^{\prime} \approx 2 \lambda(\hbar c)$. Inserting this value into (15) for $d_{\min }^{\prime}=\lambda d=0.853$, just gives $\alpha=0.69$ ).

On the other hand, the very same $\hbar \omega$ represents the energy of the meson, because all its energy is comprised in this standing wave. Hence, from (14) and (15), 
$\Phi_{0}=\frac{m_{m}^{\prime}}{8 g k_{2} d_{\min }^{\prime 2}}$

The third reason has to do with a particular relationship that follows from a general relativistic analysis presented in earlier work [9]. This states that the ratio $\Phi_{0} / \lambda$ is invariant, given by the expression,

$$
\frac{\Phi_{0}}{\lambda}=\frac{\alpha \pi \hbar c}{4\left|k_{00}\right| g d_{\min }^{\prime}}
$$

in which the quantum mechanical coupling constant $g$ is related with the electromagnetic fine constant $q_{e}^{2}=4 \pi \varepsilon_{0} g^{2} \hbar c$ and in which $k_{00}=k_{0}\left(d_{\min }^{\prime}\right)$.

This relationship (15) implies that the spacing between the two quarks remains constant, even so under excitation. The implication is that, if a pion excites into a kaon in the way as explained before, the quark spacing remains constant, while $\Phi_{0}$ and $\lambda$ assume new values under invariance of the ratio $\Phi_{0} / \lambda$ as expressed by (17). Furthermore, it has to be emphasized that the model holds for the center of mass frame. It means that quantities in this frame, such as for instance $\Phi_{0}$ and $\lambda$, assume quite different values in the lab frame, albeit under conservation of the ratio invariance. Because of the near light velocity of the meson the difference is very substantial. This consideration removes a seeming inconsistency, because on the one hand we have from (16) and (17),

$\lambda=\frac{m_{m}^{\prime}\left|k_{00}\right| \alpha \pi}{2 k_{2} d_{\min }^{\prime}(\hbar c)}, \quad$ with $m_{m}^{\prime}=m_{m} c^{2}$

while on the other hand we have (15).

This suggests as if the meson's mass is of the same order of magnitude as the rest mass value of the weak interaction boson. How to explain the discrepancy between the values of the pion's rest mass of about $140 \mathrm{MeV}$ and the weak interaction boson's rest mass of about $80.4 \mathrm{GeV}$ ? The answer is that this is an "apples and pears" comparison. The meson's rest mass is derived from its decay products in their rest mass frame, while this is not the case for the weak interaction boson (later in this article it will be argued that there is no rest frame for it). Within this view, it is not longer necessary to distinguish between off-shell and on-shell mass values and to suppose that mass conservation laws allow a violation within Heisenberg's uncertainty interval. Hence, whereas in the center of mass frame the two mass values might be about the same, it will not be longer true in the lab frame. Within the scope of this article, mesons will be described in terms of excitations from the archetype (pion). This will result in the calculation of mass ratios. It is fair to expect that the mass ratios of particles similar in nature will remain the same in the lab frame as they are in the center of mass frame. From (15) and (18) we may derive an expression for the meson's mass in the center of mass frame, as

$m_{m}^{\prime}=\left(\frac{4 d_{\min }^{\prime 2} \hbar \omega_{W}}{\alpha^{2} \pi^{2}\left|k_{00}\right|}\right) k_{2}$

It has to be emphasized once more that this expression cannot serve to calculate the lab frame value of the meson's mass in absolute terms. Nevertheless, the expression is very relevant, because it 
expresses that mass ratios can be calculated as ratios of $k_{2}\left(d^{\prime}\right)$. Taking the mass value of the pion in the lab frame as the reference, the meson's mass spectrum can be established from the behavior of $k_{2}\left(d^{\prime}\right)$.

\section{The spin-spin interaction mechanism between quarks.}

Although the simple structural model as described in section 2 seems adequate to explain the mass relationship between pions and kaons, it is not good enough to explain the full mass spectrum of mesons. One of the issues here is the difference in mass between scalar type mesons and vector type mesons with the same quark composition. Apparently, the spin orientation of the two quarks in the meson (parallel or antiparallel) has a major impact on the mass. This suggests a major influence of the spin interactions between the quarks. The life time of vector mesons is much smaller than the life time of their pseudoscalar sisters. To some extent this is comprehensible, because for similar structures, a lower state of energy is more stable than a higher state of energy. The mismatch, though, is extremely large. The reason has to do with the difference in the decay mechanism: the charged pseudoscalar mesons decay under weak interaction, while the neutral mesons and the vector mesons decay under strong interaction. Is this more than an empirical observation? Apparently, strong interaction has to do with a change in spin state, which in view of the associated life time, may occur easily. Decay under weak interaction, though, is a quantum step in the excitation mechanism of the meson structure. A symmetrical excitation brings the symmetrical composition ( $u \bar{u}$ ) into a new symmetrical composition ( $s \bar{s}$ ) with scaled quarks. This is an adiabatic process, in which the new composition is reached via an intermediate asymmetrical step, in which only one of the two quarks is scaled. This means that in between $u \bar{u}$ and $s \bar{s}$, two additional configurations may show up, to be denoted as $u \bar{s}$ and $\bar{u} s$. The excitation mechanism applies to the meson's pseudoscalar states. The energy attribution to masses of constituent quarks under regard of the influence of the energy associated with spin-spin interaction determine the mass difference with the vector-type states.

The in-depth spin-spin interaction analysis, made in the Appendix, shows that an anti-parallel alignment of the two spins in a meson increases the binding energy of the quark junction. This results into a decrease of the energy equivalent of the meson's mass by an amount of $3 A_{m}^{\prime}$, while a parallel alignment of the spin reduces the binding energy such that the energy equivalent of the meson's mass raises by $A_{m}^{\prime}$. This, as a result of the potential energy between the nuclear equivalents of magnetic moments associated with the in-product of parallel spins and anti-parallel spins, given, respectively as [7, p.172],

$\sigma_{1} \cdot \sigma_{2}=\frac{\hbar^{2}}{4} \quad$ and $\quad \sigma_{1} \cdot \sigma_{2}=-\frac{3 \hbar^{2}}{4}$

The interaction mechanism between the spins of quarks is the same as the well-known interaction between the angular momentum the proton (and its associated magnetic moment) and the anomalous angular momentum of the electron (and its associated anomalous magnetic moment) in the Hydrogen atom, which gives rise to the $21 \mathrm{~cm}$ line in its cosmological electromagnetic spectrum. In our case, these spins are the angular dipole momenta of the quarks. Hence, similarly as in electromagnetism, the interaction between these dipole momenta influence the energetic state of the mesons as just described.

Based on this analysis, a mass formulae table for the light meson sector can be assembled as shown in Table V. Before discussing the numerical assignment to the three parameters, $m_{u}^{\prime}, A_{m}^{\prime}$ and $m_{s}^{\prime}$, which are required to assign numerical values to the mass of these mesons, it is instructive to make 
some observations beforehand. Note that the pseudoscalar counterpart of the $\varphi$ meson cannot show up because of the isospin constraint. Therefore, such a meson does not exist. On the other hand, there are two well known mesons in the light sector present without any explanation in present theory. These are the $\eta$ meson $\left(549 \mathrm{MeV}\right.$ ) and the $\eta^{\prime}$ meson $(958 \mathrm{MeV})$. The energetic value of the mass of the latter one gives a fair correspondence with the energy gained by second level excitation of the archetype meson (pion). This level, however, does not match with the one from a conventional pseudoscalar $s \bar{s}$ meson, because (a) its existence is forbidden by the isospin constraint and (b) the energy level would be different as a consequence of the influence of the spin-spin interaction energy. Nevertheless, its existence is a consequence of the theory as developed in this article, because there is no reason why the pion cannot be excited into its second level. Calculation of this level gives a fair fit with the $\eta^{\prime}$ meson indeed. It is an extraordinary meson, somewhat different in structure from the straight ones. An explanation for the origin of the other extraordinary one, i.e., the $\eta$ meson, will be given in the next section.

Table V: Mass formulae for mesons in the light sector

\begin{tabular}{|l|c|c|l|l|l|l|l|}
\hline excit. & & $\mathrm{ms}$ & pseudoscalar & mass & $\mathrm{ms}$ & vector & mass \\
\hline $\begin{array}{l}\text { ground } \\
\text { state }\end{array}$ & $u \bar{u}$ & $\pi$ & $2 m_{u}^{\prime}-3 A_{m}^{\prime}$ & $\begin{array}{l}140 \\
(138)\end{array}$ & $\rho$ & $2 m_{u}^{\prime}+A_{m}^{\prime}$ & $\begin{array}{l}780 \\
(775)\end{array}$ \\
\cline { 2 - 8 } & $\eta$ & $? ?$ & $(549)$ & $\omega$ & $2 m_{u}^{\prime}+A_{m}^{\prime}$ & $\begin{array}{l}780 \\
(783)\end{array}$ \\
\hline $\begin{array}{l}\text { first } \\
\text { level } \\
\text { (strange) }\end{array}$ & $u \bar{s}$ & $\mathrm{~K}$ & $m_{u}^{\prime}+m_{s}^{\prime}-3 \frac{m_{u}^{\prime 2}}{m_{u}^{\prime} m_{s}^{\prime}} A_{m}^{\prime}$ & $\begin{array}{l}484 \\
(496)\end{array}$ & $\mathrm{K}^{*}$ & $m_{u}^{\prime}+m_{s}^{\prime}+\frac{m_{u}^{\prime 2}}{m_{u}^{\prime} m_{s}^{\prime}} A_{m}^{\prime}$ & $\begin{array}{l}896 \\
(892)\end{array}$ \\
\cline { 2 - 8 } & $s \bar{s}$ & $\eta^{\prime}$ & $? ?$ & $(958)$ & $\varphi$ & $2 m_{s}^{\prime}+\frac{m_{u}^{\prime 2}}{m_{s}^{\prime 2}} A_{m}^{\prime}$ & $\begin{array}{l}1032 \\
(1020\end{array}$ \\
\hline
\end{tabular}

Figure 3 shows these mass relationships even better. The red line in the figure shows the excitation of the pion toward the kaon and toward the $\eta^{\prime}$ meson. The blue lines show the impact of the spinspin interaction on the pion on the one hand and the $\rho$ meson and the $\omega$ meson on the other hand. It relates the mass value of the pion with the mass of the constituent $u / d$ quark. The green lines show the impact of the spin-spin interaction on the kaon and the $\mathrm{K}^{*}$ meson. It relates the mass value of the kaon with the mass of the constituent $s$ quark. The pink line shows how the mass of the vector meson $\varphi$ is determined from two constituent $s$ quarks. The vertical black axis is the mass axis. The red vertical axis is the energy axis. The offset between the two is the binding energy represented by the dotted line (as shown in Table I, a mass value 1 corresponds with an energy level 0.34). 


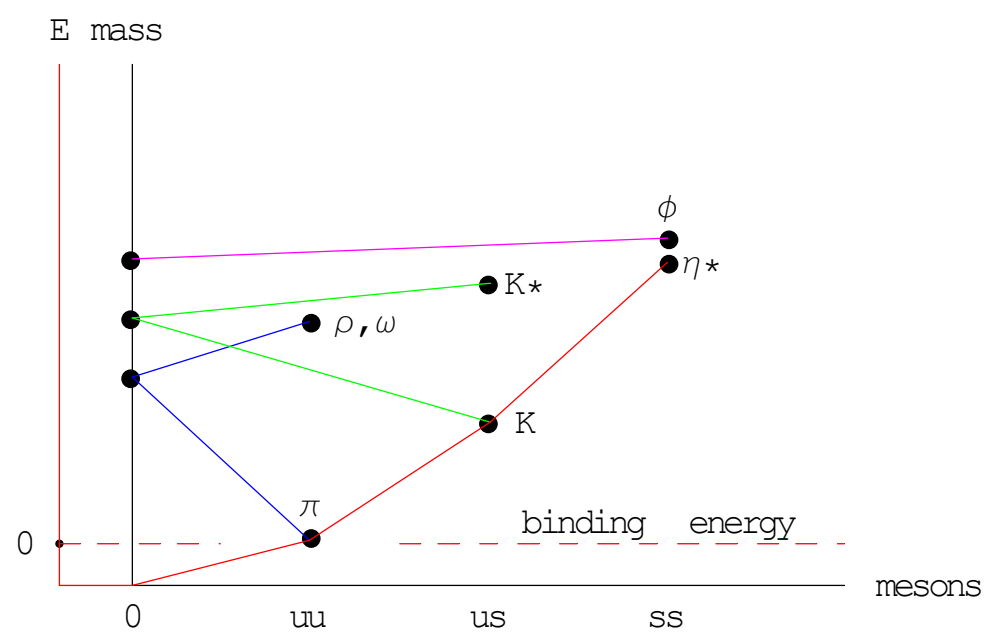

Figure 3. Illustration of the excitation mechanism of mesons and the scaling mechanism of quarks in the light sector.

Let us now discuss the assignment of numerical values to $m_{u}^{\prime}, A_{m}^{\prime}$ and $m_{s}^{\prime}$, which are used to assemble Table $\mathrm{V}$. It will be clear that three relationships are required to do so. We use,

$$
\begin{aligned}
& 2 m_{u}^{\prime}-3 A_{m}^{\prime}=m_{\pi}^{\prime}=140 \mathrm{MeV} . \\
& m_{K}^{\prime}=m_{u}^{\prime}+m_{s}^{\prime}-3 \frac{m_{u}^{\prime 2}}{m_{u}^{\prime} m_{s}^{\prime}} A_{m}^{\prime}=3.57 m_{\pi}^{\prime}=489 \mathrm{MeV} . \\
& A_{m}^{\prime}=0.51 m_{u}^{\prime}
\end{aligned}
$$

Choosing the rest mass value of the pion $\left(m_{\pi}^{\prime}=140 \mathrm{MeV}\right)$ as a reference is the first of these. The second one is taken from the excitation ratio (3.57) from pion to kaon, such as shown in Table I. The third one follows from the spin-spin analysis as presented in the Appendix. The solution of the three equations yields $m_{u}^{\prime}=300 \mathrm{MeV}$ and $m_{s}^{\prime}=478 \mathrm{MeV}$. With these values, the calculated mass equivalents of the light sector mesons $(u, s)$ show a nice match with experimental evidence, as shown in Table $V$ (the values between brackets are the actual values). The result confirms that the excitation mechanism is controlled by weak interaction

At this point, It has to be emphasized that the interaction energy $A_{m}^{\prime}=0.51 m_{u}^{\prime}$ shown in (21c), is the result of a numerical fit, taken the kaon's mass for granted and just based upon Zakharov's hypothesis that spin-spin interaction is the cause of the mass difference between the pseudoscalar mesons and the vector-type mesons. So far, we have given a theoretical basis for the kaon's mass. The additional challenge is to provide for a theoretical basis for (21c). This is covered in the Appendix. It is found that,

$$
A_{m}^{\prime}=A m_{u}^{\prime} ; A=\frac{2}{3+m_{W}^{\prime} / m_{Z}^{\prime}}
$$

Hence, inserting the known values $m_{W}^{\prime}=80.4 \mathrm{GeV}$ and $m_{Z}^{\prime}=91.2 \mathrm{GeV}$,

$A_{m}^{\prime}=0.515 m_{u}^{\prime}$ 
This is pretty close to the empirical value. It gives substantial support to the viability of the structural model shown in figure 2.

In the next section the charmonium sector $(c, s, u)$ and the bottomium sector $(b, c, s, u)$ will be discussed.

\section{The hadronization limit}

It is tempting trying to establish a theoretical value for the constituent charmed quark by considering it as the pion's third excitation mode. There is, however, no clear experimental evidence that such a meson shows up indeed. The reason of it is due to the lack of binding energy to sustain it. This can be explained as follows. As a result of excitation, the two quarks in the meson occur in a state of higher vibration. As discussed before, this is energetically equivalent with the ground state energy of two heavier quarks. It is energetically equivalent as well with the two quarks with a smaller spacing while still being in the ground state of vibration. We shall denote a meson in this condition as a meson under stress. The picture shown in figure 4, as calculated from (4)-(7), gives the illustration. The upper curve shows the increase of the meson's energy under stress, i.e. as a function of reduced spacing $d^{\prime}$. This is the curve $k_{2}\left(d^{\prime}\right)$ relative to $k_{2}\left(d_{\text {min }}^{\prime}\right)$ as a function of the half spacing $d^{\prime}$ between the quarks. From (19) it is obvious that this ratio is a mass ratio as well. The lower curve in the graph shows the binding energy as expressed by $k_{0}\left(d^{\prime}\right)$ between the quarks as a function of the spacing parameter $d^{\prime}$. For values $d^{\prime}<0.56$ the binding energy has no longer a negative value, while the spacing of the excited meson would require so. All together, the excitation mechanism can be captured by

$$
\begin{aligned}
& \frac{m_{e x c}}{m_{\pi}}=\frac{k_{2}\left(d^{\prime}\right)}{k_{2}\left(d_{\text {min }}^{\prime}\right)}=\frac{E_{1,2}^{\prime}\left(d_{\min }^{\prime}\right)-E_{\text {bind }}^{\prime}\left(d_{\text {min }}^{\prime}\right)}{E_{0}^{\prime}\left(d_{\text {min }}^{\prime}\right)-E_{\text {bind }}^{\prime}\left(d_{\text {min }}^{\prime}\right)}, \text { where } d^{\prime} \leq d_{\text {bind }}^{\prime} \text {, and } \\
& E_{\text {bind }}^{\prime}\left(d_{\text {bind }}^{\prime}\right)=0 .
\end{aligned}
$$

This expression limits the excitation mode as discussed so far. It is the light sector limit. It is determined by the equivalence of the vibration energy in the first, respectively, the second excitation mode at the spacing of minimum energy $\left(d_{\text {min }}^{\prime}\right)$ and the vibration ground state energy at a somewhat reduced value of the normalized spacing $\left(d^{\prime}=d_{0}^{\prime}=d_{0} \lambda\right)$. Keeping in mind that the absolute value of the spacing is kept constant by the wavelength of the weak interaction boson, it implies that the $\lambda$-value of the meson is modified, say to $\lambda^{\prime}$, and, because of the ratio invariance $\Phi_{0} / \lambda$, the $\Phi_{0}$ - value is modified as well. As a consequence we have $d_{\min } \lambda=d_{0} \lambda^{\prime}=d_{\min }^{\prime}$. This mechanism creates the kaon and the $\eta^{\prime}$ mesons as well as the mesons associated with those as a consequence of the spin-spin interaction. It means that a meson under stress is converted into a unstressed meson with scaled quarks. 


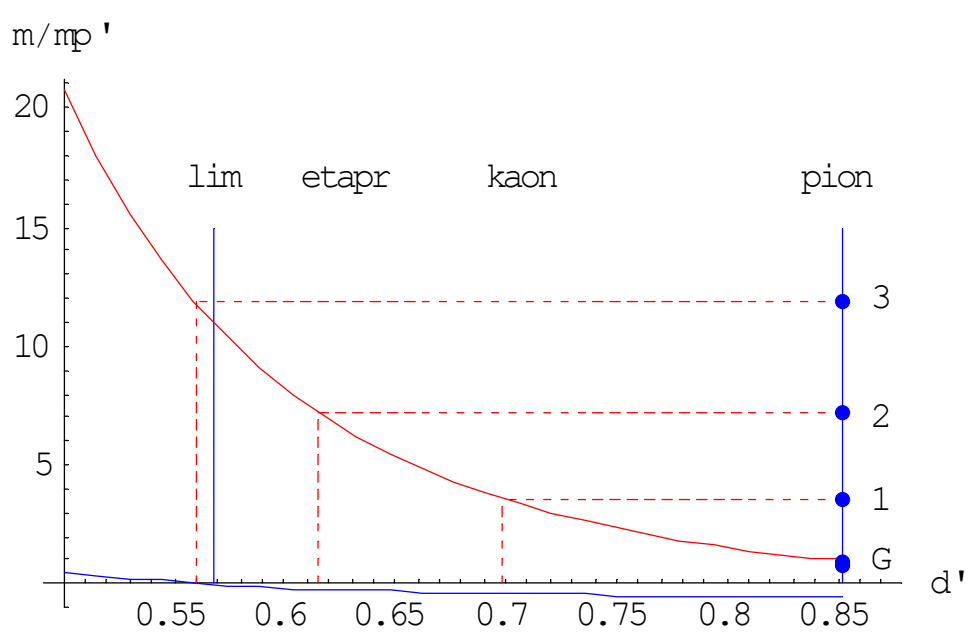

Figure 4. The light sector limit. The graph shows the increase of the massive energy of a quark/antiquark pair relative to the pion state as a function of the quark spacing. Two excitation levels beyond the pion's ground state are converted into the ground state of, respectively, the kaon and the $\eta^{\prime}$, thereby producing the $(u, s)$ - quark family. Third level excitation is prevented by the loss of binding energy (lower curve).

This is not the end of the story. Further excitation is possible under bootstrapping by regarding the $s \bar{s}$ - meson as an upscaled meson. Continuation under first level excitation scales $s \bar{s}$ towards $c \bar{c}$ (charmonium) and $c \bar{c}$ towards $b \bar{b}$ (bottomium). The scaling factor is expected to be the same as the scaling factor from pion to kaon, i.e. about 3.54. However, the mass ratio of $J / \psi(=c \bar{c})$ over $\varphi(=s \bar{s})$ is about 3 and the same holds for the mass ratio of $Y(=b \bar{b})$ over $J / \psi(=c \bar{c})$ as well. Apparently, there is a difference between the light sector and the bootstrapped ones. This difference has to do with the binding force. As we have seen, excitation decreases the binding force. So, if the excitation starts again with upscaled quarks in the charmonium sector in a bootstrap condition similar to that of the pion, how to explain the associated binding energy? The answer is that this negative amount of energy is compensated by some (positive) massive energy gained by the meson. This requires an adaptation of the mass formulae, such that for pseudoscalar mesons,

$$
m_{i}^{\prime}+m_{c}^{\prime}-3 \frac{m_{u}^{\prime 2}}{m_{i}^{\prime} m_{c}^{\prime}} A_{m}^{\prime} \rightarrow m_{i}^{\prime}+m_{c}^{\prime}+\left(\delta_{c} \frac{m_{u}^{\prime}}{m_{i}^{\prime}}-3 \frac{m_{u}^{\prime 2}}{m_{i}^{\prime} m_{c}^{\prime}} A_{m}^{\prime}\right)
$$

Of course, for vector mesons the factor -3 has to be replaced by +1 . The pedestal $\delta_{c}$ represents the converted binding force energy. The pedestal is zero in the light sector. This pedestal is present in Zakharov's heuristic formulae as well [3], but no explanation is given why. It is just introduced to give a better empirical fit. The presence of physical mass turns the anharmonic quantum mechanical twobody oscillator into a near harmonic one, thereby reducing the excitation factor 3.57 into about 3 , such that the masses of the constituent quarks $c, b$ are readily established as

$m_{c}^{\prime} \approx 3 m_{s}^{\prime}$ and $m_{b}^{\prime} \approx 3 m_{c}^{\prime}$.

Calculation of the meson masses in the charmonium sector and in the bottomium sector from (16) and (17) after assigning empirical values $\delta_{c}=138 \mathrm{MeV}$ and $\delta_{b}=291 \mathrm{MeV}$, shows the result as summarized in Table VII. The upper half of the table shows the PDG values, the lower half shows the calculated values. The mass values in front of the slash "/" are those of the vector mesons and those behind it are those of the pseudoscalar mesons. 
Table VII: Calculated meson masses compared with experimental evidence

\begin{tabular}{|l|l|l|l|l|l|}
\hline \multicolumn{5}{|c|}{ experimental evidence } \\
\hline$m_{u}^{\prime}$ & & $u \bar{u}(775 / 139)$ & $u \bar{u} \pm \bar{u} u$ (782/n.a.) & & \\
\hline$m_{s}^{\prime}$ & & $s \bar{s}(1020 /$ n.a.) & $s \bar{u}(892 / 489)$ & & \\
\hline$m_{c}^{\prime}$ & & $c \bar{c}(3096 / 2983)$ & $c \bar{s}(2112 / 1968)$ & $c \bar{u}(2007 / 1869)$ & \\
\hline$m_{b}^{\prime}$ & & $b \bar{b}(9460 /$ n.a.) & $b \bar{c}$ (?/6276) & $b \bar{s}(5415 / 5367)$ & $b \bar{u}(5325 / 5279)$ \\
\hline
\end{tabular}

\begin{tabular}{|l|l|l|l|l|l|}
\hline \multicolumn{7}{|c|}{ calculated } \\
\hline$m_{u}^{\prime}$ & 310 & $u \bar{u}(780 / 140)$ & $u \bar{u} \pm \bar{u} u$ (780/n.a.) & & \\
\hline$m_{s}^{\prime}$ & 483 & $s \bar{s}(1032 /$ n.a.) & $s \bar{u}(896 / 485)$ & & \\
\hline$m_{c}^{\prime}$ & 1515 & $c \bar{c}(3096 / 2983)$ & $c \bar{s}(2119 / 1988)$ & $c \bar{u}(1996 / 1865)$ & \\
\hline$m_{b}^{\prime}$ & 4720 & $b \bar{b}(9469 /$ n.a.) & $b \bar{c}(6263 / 6263)$ & $b \bar{s}(5400 / 5358)$ & $b \bar{u}(5332 / 5289)$ \\
\hline
\end{tabular}

It will be clear that the calculation gives a perfect fit with experimental evidence. It satisfies the qualitative explanation. The required empirical fine-tuning of the pedestal values is a slight shortcoming still. It is probably fair to say that this result is at least as good, if not better than obtained by any other theory based approach to give a detailed explanation of the meson's mass spectrum [16].

A major issue now is the question why the excitation mechanism would stop at the bottomium level. It would be reasonable to expect a next generation on the basis of a constituent quark of about 14 $\mathrm{GeV}\left(\approx 3 m_{b}^{\prime}\right)$. But there is no experimental evidence of such a generation. Instead, a topquark is found at the level of $175 \mathrm{GeV}$. However, not as part of hadrons. What could be the reason? As far as the author is aware of, present theory does not answer this question. Quite interestingly, within the view as presented so far in this article, it appears to be possible to explain this phenomenon. Once again, it has to do with the binding force between the two quarks. We have seen before that third level excitation from the pion state is prevented by the loss of binding energy. This has been the reason that excitation is continued by a different process, i.e., by bootstrapping as $s \bar{s} \rightarrow c \bar{c} \rightarrow b \bar{b}$. Meson scaling, however, is only possible as long as the energy won by excitation is larger than the loss in binding energy. Figure 5 shows that this may come to an end. If the spacing between stressed quarks in the basic mode becomes too small, energy won by excitation cannot longer compensate the loss in binding energy. This will happen for $d^{\prime}<d_{0}^{\prime}$, where $d_{0}^{\prime}$ is defined by

$E_{\text {bind }}^{\prime}\left(d_{0}^{\prime}\right)-E_{1}^{\prime}\left(d_{0}^{\prime}\right)=0$.

Calculation from (6) $-(13)$, illustrated in figure 5 , shows that this happens to be for $d_{0}^{\prime} \leq 0.39$. The $d_{0}^{\prime}$ - values of the charmonium $(c \bar{c})$ and the bottomium $(b \bar{b})$ appear to be, respectively, $d_{0}^{\prime}=0.49$ and $d_{0}^{\prime}=0.39$. As follows from (19), these two values can be readily established from the condition

$140 \frac{k_{2}^{\prime}\left(d_{0}^{\prime}\right)}{k_{2}^{\prime}\left(d_{\text {min }}^{\prime}\right)}=3096($ for $c \bar{c}=J / \psi)$, respectively $9460($ for $b \bar{b}=Y$ ). 


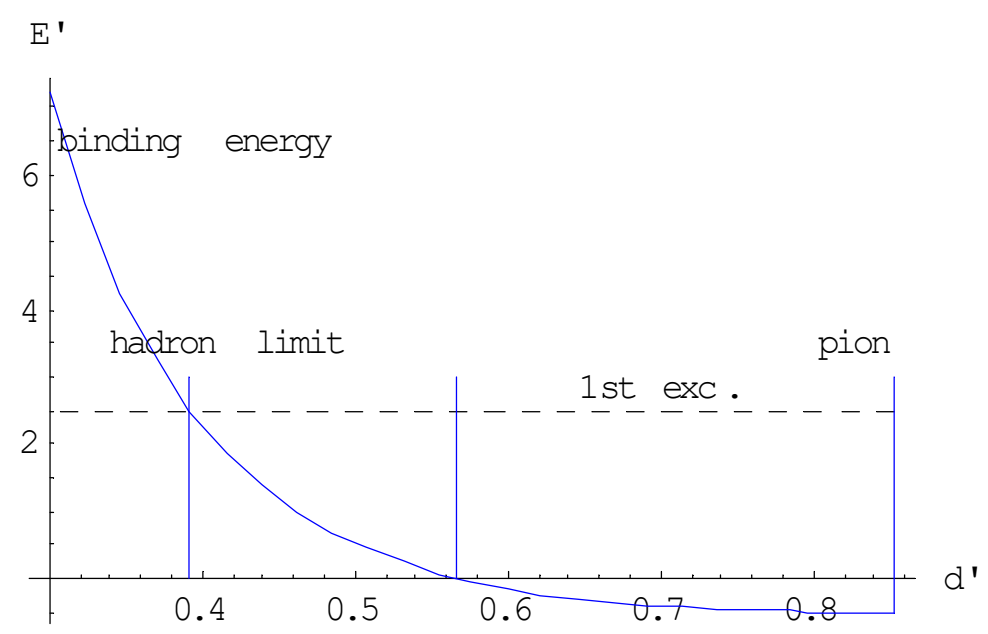

Figure 5. The hadronization limit. Bootstrapping stops as soon as the energy gained by excitation (represented by the dotted line) is not sufficient to compensate the loss in binding energy (represented by the curve showing the binding energy as a function of the quark spacing).

This means that the charmonium can excite and the bottomium cannot. Quark hadronization beyond the bottomium generation appears to be impossible. This is illustrated in figure 6 . The mass curve is the quantitative equivalent of the relative one shown in figure 4.

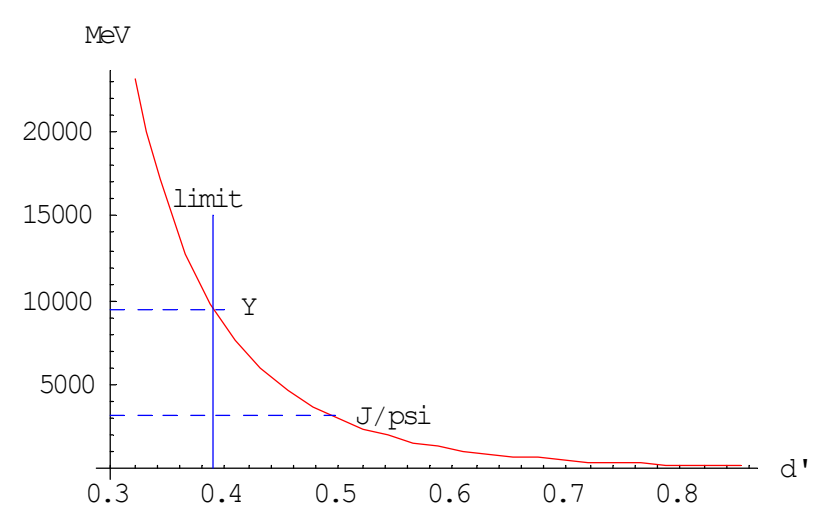

Figure 6. The quark spacing in $J / \psi$ and in $Y$, as compared with the quark spacing set by the hadronization limit.

Let us now come back on the $\eta-\eta^{\prime}$ puzzle. I have already shown that second level excitation from the pion state is possible, because of the preservation of some residual binding energy. As shown earlier, it explains the origin and the mass of the $\eta^{\prime}$ meson. The mass problem of the $\eta$ meson is left. So far, we have only discussed an excitation mechanism, in which scaled mesons are created from the equivalence of the first level excitation state vibration energy of the original meson with the ground state vibration energy of the scaled mesons. This mechanism has been illustrated in fig. 4 . Note that these vibration energies are nett energies (= binding energy not included). In the light sector, a second scaling mechanism shows up that is absent in the two higher sectors. In this particular mechanism scaling occurs by an indirect mechanism. Let us consider a meson under stress, such that gradually the spacing between the quarks is reduced. As a consequence, the gross ground state energy (with the binding energy included) raises. At a particular state of spacing, this ground state energy is equal to the first level excitation gross energy in the original unstressed state of the meson. See figure 7 (upper part). This state can straightforwardly be calculated for, e.g., the pion. It occurs at a spacing $d_{0}^{\prime}=0.552$. The corresponding state of energy of this (stressed unscaled) meson 
can be calculated as well. It can be read from fig. 5 as well. The massive energy amounts to 1741 $\mathrm{MeV}$. This state corresponds with the first level excitation state of a scaled (unstressed) one at reduced spacing. The spacing of this scaled meson can be calculated as $d_{0}^{\prime}=0.685$. It is slightly smaller than the quark spacing of a kaon $\left(d_{0}^{\prime}=0.698\right)$. It makes a new meson with a massive energy of $545 \mathrm{MeV}$, known as the $\eta$ meson!

It has to be emphasized once more that the constituent masses model the holistic behavior of the meson structure. No more, no less. They do not necessarily exist as separate identities, albeit that we have to do with two energetic kernels (and three in the baryon case).Therefore, there is no reason why the assigned mass values should show up identical to those in the baryon structure. In fact, they are somewhat different [7, p. 122]. In previous work it has been shown that the baryon structure can be analyzed similar to the meson structure [17].

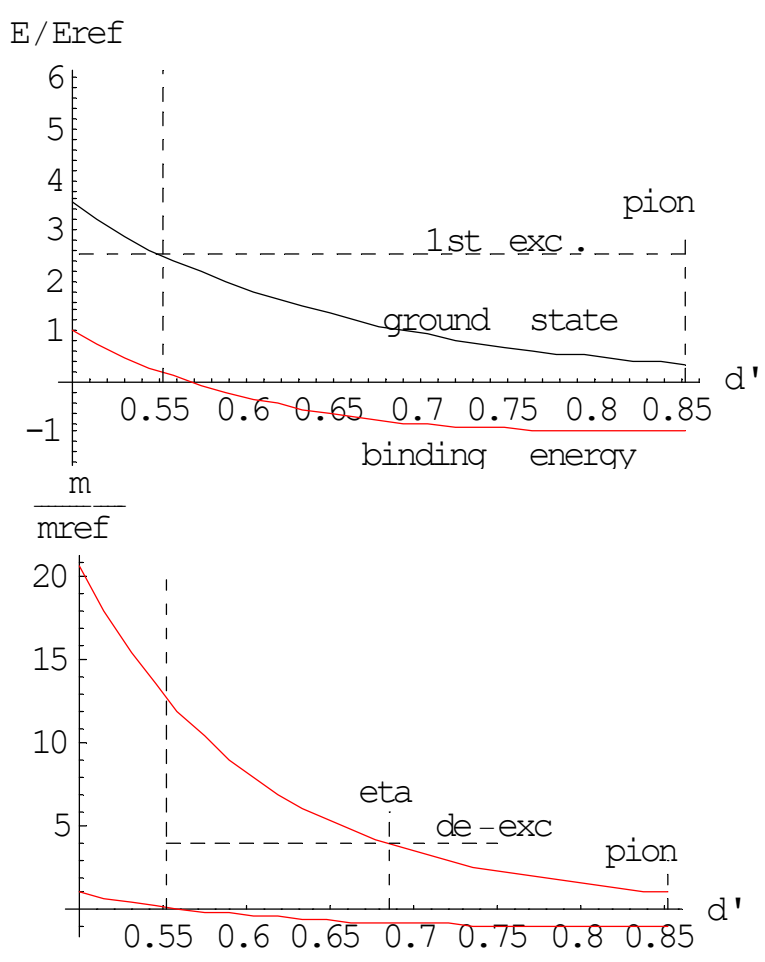

Figure 7. The origin of the $\eta$-meson. The upper diagram shows the equivalence of the ground state energy of a meson under stress with the first level of excitation of the unstressed meson. This condition cannot be maintained because the quark spacing causes a positive binding energy. The condition is subject to deexcitation towards the ground state of a scaled meson, illustrated in the lower diagram.

\section{The topquark and the nuclear bosons}

\subsection{The topquark}

In the interpretation outlined so far, the origin of $s, c$ and $b$ quarks can be readily understood, as well as their constituent mass values. The mass value of the top quark, however, is out of scale. Moreover, the absence of quarkonia beyond the bottomium, as a consequence of the bootstrap stop, prevents a continuation of the quark scaling beyond the $b$ quark. Therefore, the top quark seems belonging to a different category, as a second elementary quark next to the $u / d$ archetype. 
This evokes a question about its reason of existence. Comay [18] proposes to regard the $W / Z$ boson as a meson built by a top quark in conjunction with another quark. This would explain its origin. The difference between the mass of the $\mathrm{W} / \mathrm{Z}$ boson with twice the mass of the topquark is due to the binding energy, similarly as the difference of the mass of a pion with twice the constituent mass of the $u / d$ quark. If the second quark is an antitop quark, this proposal shows a clear fit with the pion model as expressed by (14), because rewriting (14) in terms of an elementary quark with energy $m_{t}^{\prime}$, gives

$m_{W}^{\prime}=2 m_{t}^{\prime}-3 A_{t}^{\prime}$.

With $m_{t}^{\prime}=175 \mathrm{Gev}, A_{t}^{\prime}$ should amount to $A_{t}^{\prime}=0.51 m_{t}^{\prime}$ to produce $m_{W}^{\prime}=80.4 \mathrm{GeV}$. This $A_{t}^{\prime}$ value for topquark mesons show a clear correspondence with the $A_{m}^{\prime}$ value $\left(=0.51 m_{u}^{\prime}\right)$ for the mesons, shown by (21c). This is consistent with its independence from the $u / d$ quark.

This view suggests a hierarchical system, such as illustrated in figure 8 . The intrameson boson is the virtual $\mathrm{W} / \mathrm{Z}$ between the quark and the antiquark. Once the virtual $\mathrm{W} / \mathrm{Z}$ becomes free (similarly as a virtual photon does by escape from an atomic shell), it can be described as a topquark-antitop bond. The interaction between two baryons is similar. The scalar fields from the three baryon quarks extend beyond the boundaries of the baryon. The external ("remnant") baryon field, observed as a "far field", has a similar format as the quark field [9]. Therefore, the bond between two baryons can be described similarly as the bond as the quark-antiquark bond in a meson. Only, the coupling factor of the baryons to this external baryon field is different from the coupling factor of the quarks to the intra-meson field. It shows similar characteristics. The bond between the baryons is a virtual meson. Once it becomes free, it can be identified as a real meson. So, whereas the internucleon boson in real state is a meson (built up by a quark-antiquark), the intra-meson boson in real state is the W/Z built-up by a top quark-antitop quark. In both cases the boson field is Higgs-type, characterized by the $\Phi_{0} / \lambda$ invariance.

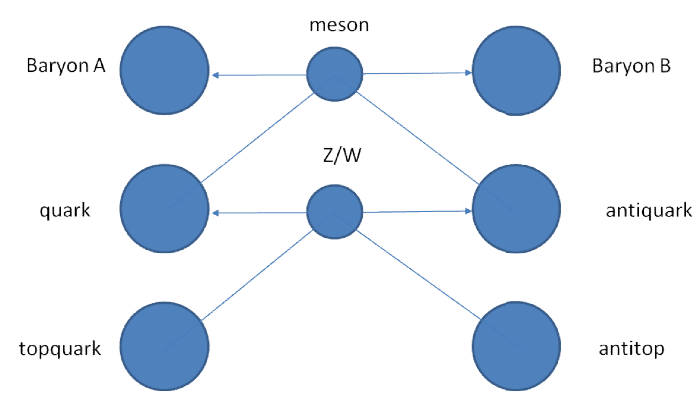

Figure 8. Meson hierarchy. The quantum of the bosonic field between two baryons is a meson in virtual state. The meson in real state is a quark-antiquark assembly bound by $W / Z$ bosons in virtual state. The $W / Z$ in real state behaves as mesonic topquark-antitop bond.

There is a problem, though. A top-antitop composition of a meson would produce a neutral meson. So, it fits for the $Z$, but it does not for the $W$ (a reason for Comay to hypothesize a nonhomogeneous quark composition for his $W / Z$ meson [18]). If the topquark would have an isospin sister, the problem would disappear. Conventionally, however, isospin is exclusively associated with $u / d$. But, having seen now that $u, d, s$ and $c$ are members of a scaled family of the archetype quark and that there are reasons to consider the topquark as a second elementary quark (instead of a sixth 
one), it is fair to criticize isospin as a dogma for $u / d$ only. It is true that there is no proof of isospin associated with the topquark. On the other hand, there is no disproof either, because the decay paths of the topquark are not incompatible with such a hypothesis (neither the top quark, nor the $\mathrm{W} / \mathrm{Z}$ bosons are observables: their experimental signatures are explained with a theory in mind).

\subsection{The nuclear bosons}

In the preceding paragraphs it has been demonstrated that the meson's mass spectrum can be explained from the quark's far field as defined in (2), supplemented by a near field from a dipole moment. The far field is the steady state solution of a wave equation with the format

$$
\frac{1}{c^{2}} \frac{\partial^{2} r \Phi}{\partial t^{2}}-\frac{\partial^{2}}{\partial r^{2}} r \Phi+\lambda^{2} r \Phi=\rho_{H}(r, t)
$$

where $\rho_{H}(r, t)$ is a Dirac-type pointlike source that can be expressed as,

$\rho_{H}(r, t)=4 \pi \frac{\Phi_{0}}{\lambda} \delta^{3}(r) H(t)$,

in which $H(t)$ is Heaviside's step function. Its solution is given by [19],

$$
r \Phi(r, t) \leftrightarrow \frac{\Phi_{0}}{\lambda} \frac{1}{s} \exp \left[-\left(\lambda r \sqrt{\left.s^{2} /(\lambda c)^{2}+1\right)}\right]\right.
$$

The steady state solution of (30) is similar to a shielded Coulomb field as, for instance, like the field of a pointlike charged particle in a plasma, such as described for the first time by Debije [20]. It can be derived from a Lagrangian as in (8) where the field potential is given by,

$$
U(\Phi)=-\frac{1}{2} \lambda^{2} \Phi^{2}
$$

Such a Lagrangian is the scalar part of the more general Proca Lagrangian [9]. It is also the far field part of the Higgs field (9), where $|\Phi|<<1$, which corresponds with $\lambda r>>1$.

Equation (30) is known as well as the Klein-Gordon equation, which is considered as the (scalar) wave equation of a bosonic spin-0 particle. The right-hand part of (31) is a Laplace transform. Its conversion into the time domain requires a numerical solution, given in [21]. The transient part is a propagating rapidly decaying pulse, as shown in figure 9 . Such a pulse is the result of the annihilation of a quark with an antiquark. As a consequence of the dispersive character, the various frequency components in the pulse are dying out in different ways. Dispersion is equivalent with a change of pulse propagation at light speed into propagation at group velocity with "near light speed". That marks the difference with the gamma-photons in the annihilation process of an electron and a positron. In both cases it is impossible to assign a rest frame to the bosonic particle. It is shown in figure 9 that the flux $\Phi$ may assume negative values, while it is is expressed in units of energy, e.g. in $\mathrm{MeV}$. This is somewhat contra-intuitive. It has to be taken into account, though, that energetic flux is not the same as energy, because energy is built up by the square of the flux and normalized on a reference value with energy metric (see Appendix I of [6]).

As explained before in [6], the energy of this boson, be it interpreted as a Proca one or a KleinGordon one, is different from physical mass, because there is neither a mass term in the Lagrangian 
density, nor in the solution of the wave equation as shown by (31). There is a $\lambda-$ term, which specifies the spatial range of the nuclear force, which is in Proca's original formulation usually interpreted as a physical mass term $\lambda=m c^{2} / \hbar c$ (Note that this is consistent with (31), because of the resonance $\hbar \omega=\lambda c$ ). However, owing to the invariance of the ratio $\Phi_{0} / \lambda$, the energy of the far field Proca boson remains, similar as a photon, the same in any inertial frame. If somebody tries to bring the Proca boson to rest, the $\lambda$ - term changes in coherence with the change of $\Phi_{0}$.

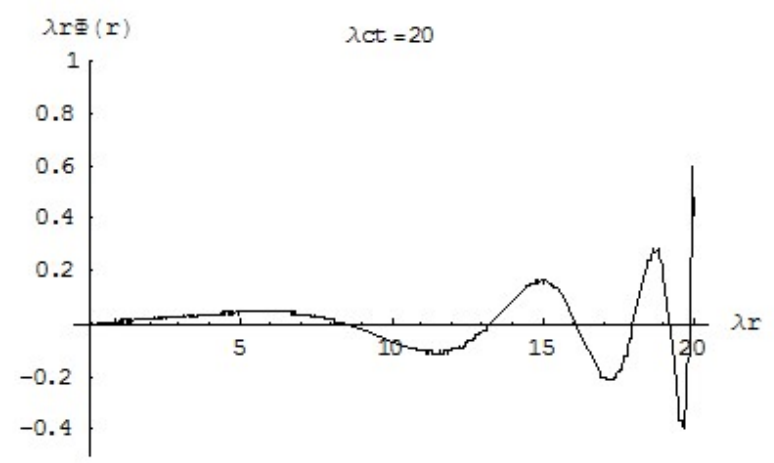

Figure 9. Propagation of a Proca boson after annihilation of a quark and an antiquark.

If, under violence of particle collisions, the equilibrium between the quarks is broken, the far field bosons as well as the near field bosons will show up in decay channels of pairs of gamma photons, W-bosons or Z-bosons, which will manifest themselves into a decay path of fermions. Momenta and energies of these fermions can be measured and can be traced back to numerical values for the energy of two nuclear bosons pairs. So, ultimately, the Higgs field will show up as two quantum fields, instead of the single one that is expected by the Standard Model. The massive energy of the far field part, if interpreted as a single boson, would therefore be assigned as,

$m_{H}^{\prime} \approx 2 \lambda(\hbar c)$.

Subsequent application of (15) on this gives,

$m_{H}^{\prime}=\frac{4 d_{\min }^{\prime} m_{W}^{\prime}}{\alpha \pi}=127 \mathrm{GeV}$

which nicely fits with experimental evidence from the detection in 2012 by CERN of a $125 \mathrm{GeV}$ bosonic particle. Note that this detection is an interpretation of the energy of a diphoton bump. Interpreting these photons as decays of the bosons as a consequence of the confinement break quark and antiquark, like I propose here, is a valid alternative for the conclusion that the source of this diphoton bump are two bosons. It will be clear that the view as presented in this article shows an intimate relationship between the $80.4 / 90 \mathrm{GeV}$ W/Z weak interaction particles, the scalar $125 \mathrm{GeV}$ Higgs particle and the $175 \mathrm{GeV}$ topquark. 


\section{Conclusions}

The identification of the archetype quark as a Dirac-particle with an anomalous linear dipole moment next to the well-known anomalous angular dipole moment reveals an intimate relationship between particles that in present-state theory are regarded as elementary. The nuclear bosonic field spread by the archetype quark can be profiled by a two-parameter expression, one parameter for the strength ( $\Phi_{0}$ in $\mathrm{eV}$ ) and one for the spatial reach $\left(\lambda\right.$ in $\left.\mathrm{m}^{-1}\right)$. A previous study [9] has shown that the ratio $\Phi_{0} / \lambda$ is a frame-independent constant with a value that can be established by theory. This nuclear bosonic field is made-up as the sum of the dipole field next to a classical field shielded by an uniformly distributed background field. The particles considered within the scope of this article fall apart into two families, namely a (relative) low energetic one and a (relative) high energetic one. The low energetic family comprises the $u / d, s, c$ and $b$ quarks and the high energetic family comprises the $W / Z$ "bosons", the topquark and the "Higgs boson" responsible for the background field. The difference between the two families is a difference in the quark strength parameter $\Phi_{0}$ (or, if preferred, in the quark reach parameter $\lambda$ ). It means that a single particle from each of the two families may serve as a true elementary particle. The other ones will no longer be elementary.

It has been shown in this article that all mesons show up as excitations of the archetype pion. The energetic values of these excitations determine the rest mass values of the mesons. These values are established by numerical calculations on an anharmonic 2D oscillator model, thereby taking into account the bosonic spin-spin interaction caused by the dipole fields (similarly as happens in the hyperfine structure of the hydrogen atom as a consequence from the interaction between the spins of electron and proton). Whereas the mesons show up as excitations, the constituent quarks in these mesons show up a scaled variants of the basic $u / d$ archetype.

The side-effect of the excitation mechanism is a loss in binding energy. It has been shown that after three meson generations, i.e. the basic quarkonium with $u . / d, s$ quarks, the charmonium with $u . / d, s, c$ quarks and the bottomium with $u . / d, s, c, b$ excitation under negative binding energy cannot longer occur. Hence, upscaling from the archetype $u / d$ quark no longer takes place. This means that the origin of the topquark is different. This is not surprising because of the large mismatch between the rest mass of this quark as compared to the rest masses of the $u / d$ quark family. Application of the knowledge gained by the study of the spin-spin interaction mechanism has revealed that the topquark and the antitop can be interpreted as constituent quarks of the $\mathrm{W} / \mathrm{Z}$ bosons conceived as mesons.

\section{Discussion}

It may seem that the theory as developed in this article, and before in previous work [9], is an alternative one, different from, or even in conflict with, the present-state theory as summarized in the Standard Model. That it is in conflict, is not per se necessarily true. What is true, however, is that the structure-based theory as described in this article is developed by choosing an angle different from that in the mathematical oriented view as adopted in the Standard Model. Judging the merit of an alternative theory requires answering of three basic questions. These are "Is the theory consistent"? "Is it in agreement with experimental evidence"? and "Does it bring something new"? In quantum physics, there is a difficulty with the second of these questions. Experimental data on observables (like many fermions) are "hard", but experimental data on non-observables, like bosons, are "soft". They show up as "signatures", which are interpreted with a theory in mind. Therefore, some of the existence proofs of non-observables are theory-dependent. Hence, some care is required in judging the agreement with experimental data. The Standard Model is considered a well 
proven theory. That means that it is consistent with its axioms and that the results from the theory are in agreement with experimental evidence. The axioms in QCD are the $S U(n)$ formalism and the color force assignment to quarks. Choosing a different angle may imply a reversal of argumentation. Rather than considering results as the consequences of axioms, the opposite might be true: the consequences might be the reasons for the existence of the axioms. To prove that it might be useful to reverse the argumentation should be justified by showing that the change of view brings something new. I believe that the conclusions summarized in the preceding section do so. Interpreting isospin and the intimate gluing of quarks in hadrons as a consequence of Dirac's "forgotten" second dipole moment might be just an alternative interpretation for the gluons that pop up in the $\mathrm{SU}(\mathrm{n})$ formalism. It is true that the explanation of the origin of mass requires the existence of an energetic background, just similar to the one as to be adopted in cosmology, but there is no reason why this field should be subject to a kind of spontaneous symmetry breaking if Dirac's second dipole moment is taken into account. Doing the latter has the clear benefit that the number of elementary particles can be drastically reduced. And so on.

The view as outlined in this article only covers a part of particle physics. Baryons have not been discussed and decay processes have not been considered. A previous study has shown, however, that baryons can be modeled as the one-body equivalent of a three-body mechanical oscillator, in a similar way as the one-body equivalent of a two-body oscillator model for mesons [21]. It will not be a hard task to include the spin-spin interaction mechanism as developed in this article. It is fair to say that proven theories do not exist. A theory can be consistent with its axioms. A theory can be in agreement with experimental evidence. The more axioms, the more accurate a theory will be. In this respect, the present Standard Model has obtained a rather mature state. Nevertheless, reducing the number of axioms may pave the way for deeper understanding and answering unsolved problems. It is my belief that the theory as outlined in this paper, is not necessarily in conflict with the achievements of the Standard Model, albeit that an underlying physical level is added. More on this can be found in [22], which contains a fundamental discussion and comparison of the gauge-based Standard Model on the one hand (with axiomatic gauges) and the structural view as described so far in this article on the other hand (with structure based gauges). Finally, I wish to express my belief as well that the mass relationships as developed in this article, are not in conflict with the achievements of the Particle Data Group (PDG).

\section{Appendix A: The interactions between the spins and the isospins in a meson}

Similarly as the magnetic moment of the electron in its orbit around a proton or positron interacts with the magnetic moment of the proton or positron, the nuclear moments of the quark interact. For its analysis, I'Il assume Griffith's model as a starting point. In Griffith's model, the interaction energy $U_{12}$ between an electron and the proton nucleus of the hydrogen atom amounts to [23],

$U_{12}=\mu_{0} \frac{\gamma_{g 1} \gamma_{g 2} e^{2}}{6 m_{1} m_{2}} \frac{\left(\boldsymbol{\sigma}_{1} \cdot \boldsymbol{\sigma}_{2}\right)}{\pi d_{0}^{3}}=\frac{4}{6 c^{2}} \frac{e^{2}}{4 \pi \varepsilon_{0}} \frac{\gamma_{g 1} \gamma_{g 2}}{m_{1} m_{2}} \frac{\left(\boldsymbol{\sigma}_{1} \cdot \boldsymbol{\sigma}_{2}\right)}{d_{0}^{3}}$

in which $\mu_{0}$ and $\varepsilon_{0}$ are the Maxwellian permeability constants, $\gamma$ 's the gyromagnetic ratios, $m$ 's the rest masses and in which $d_{0}$ is radius of the orbit. The quantities $\sigma_{1}$ and $\sigma_{2}$ are spin vectors with eigen value $\hbar / 2$. To establish the nuclear equivalent of the electromagnetic potential, the far field force $F_{F}$ evoked by a quark is compared with the electromagnetic force $F_{e}$. Generally, under consideration of (3), 


$$
F_{e}=-e \frac{\partial}{\partial r} \frac{e}{4 \pi \varepsilon_{0} r} \quad \text { and } \quad F_{F}=-g \frac{\partial}{\partial r} \Phi_{0} \frac{\exp (-\lambda r)}{\lambda r}
$$

There is no reason why these forces would be the same. What is clear, however, is, that $g \Phi_{0} / \lambda$ plays a similar role as $e^{2} /\left(4 \pi \varepsilon_{0}\right)$, i.e.,

$$
\frac{e^{2}}{4 \pi \varepsilon_{0}} \leftrightarrow \frac{g \Phi_{0}}{\lambda}
$$

Hence, from (A-1) and (17) we have for two identical particles $m_{q u}$ in the pion,

$$
U_{12}=\frac{1}{c^{2}} \frac{4}{6} \frac{e^{2}}{4 \pi \varepsilon_{0}} \frac{\gamma^{2}}{m_{q u}^{2}} \frac{\left(\boldsymbol{\sigma}_{1} \boldsymbol{\sigma}_{2}\right)}{d_{0}^{3}} \rightarrow \frac{1}{c^{2}} \frac{2}{3} \frac{g \Phi_{0}}{\lambda} \frac{\gamma^{2}}{m_{q u}^{2}} \frac{\left(\boldsymbol{\sigma}_{1} \boldsymbol{\sigma}_{2}\right)}{d_{0}^{3}}
$$

The spins will align themselves in parallel or in anti-parallel, which gives, respectively,

$\boldsymbol{\sigma}_{1} \cdot \boldsymbol{\sigma}_{2}=\frac{\hbar^{2}}{4} \quad$ and $\quad \boldsymbol{\sigma}_{1} \cdot \boldsymbol{\sigma}_{2}=-\frac{3 \hbar^{2}}{4}$

Hence, the energy difference between the parallel spin condition and the antiparallel condition of the quarks in the meson is given by,

$$
\Delta U_{q u}=\frac{2}{3 c^{2}} w \frac{g \Phi_{0}}{\lambda} \frac{\gamma_{g}^{2}}{m_{q u}^{\prime 2}} \frac{\hbar^{2}}{d_{\min }^{3}}=\frac{2}{3 c^{2}} w\left(g \Phi_{0}\right) \frac{\gamma_{g}^{2}}{m_{q u}^{\prime 2}} \frac{(\hbar c)^{2}}{d_{\min }^{\prime 3}} \lambda^{2},
$$

in which $w$ is the far field weighing factor as defined in (3).

Invoking (32) and (15) reveals the relationships,

$$
\begin{aligned}
& g \Phi_{0}=\hbar \omega_{W}=m_{W}^{\prime}, \\
& \lambda=\frac{m_{H}^{\prime}}{2 \hbar c},
\end{aligned}
$$

In which $m_{W}^{\prime}$ and $m_{H}^{\prime}$ are the energies of, respectively, the weak interaction boson $W$ and the Higgs boson. Applying (A-7) and (A-8) to (A-6) gives,

$$
\Delta U_{q u}=\frac{2}{3} w\left(\hbar \omega_{W}\right) \frac{\gamma_{g}^{2}}{m_{q u}^{2}} \frac{(\hbar c)^{2}}{d_{\min }^{\prime 3}}\left(\frac{m_{H}^{\prime}}{2 \hbar c}\right)^{2}
$$

It has to be taken into account that $m_{q u}^{\prime}$ represents the energy of the quark masses $m_{q u}$ in the center-of-mass, while the bosons $\hbar \omega_{W}$ and $m_{H}^{\prime}$ are known as relativistic energies. A proper lab frame interpretation of $(A-9)$ requires a relativistic correction of these energies. This is possible under 
consideration of the view that $\hbar \omega_{W}$ represents the rest mass energy of the pion at relativistic speed. This view enables a relativistic correction of (A-9) from $\Delta U_{q u}$ into $\Delta U_{\pi \rho}$, such that

$$
\begin{aligned}
& \Delta U_{\pi \rho}=\frac{1}{6} w \frac{\gamma_{g}^{2}}{d_{\min }^{\prime 3}}\left(\frac{m_{H}^{\prime}}{m_{W}^{\prime}}\right)^{2}\left(\frac{m_{\pi}^{\prime}}{m_{q u}^{\prime}}\right)^{2} m_{\pi}^{\prime}=B\left(\frac{m_{\pi}^{\prime}}{m_{q u}^{\prime}}\right)^{2} m_{\pi}^{\prime} ; \\
& B=\frac{1}{6} w \frac{\gamma_{g}^{2}}{d_{\min }^{\prime 3}}\left(\frac{m_{H}^{\prime}}{m_{W}^{\prime}}\right)^{2} .
\end{aligned}
$$

The magnitude of the dimensionless factor $B$ can be quantified from $w=1 / 0.55, \gamma_{g}=2, d_{\min }^{\prime}=$ 0.856, $m_{H}^{\prime}=127 \mathrm{GeV}$ and $m_{W}^{\prime}=80.4 \mathrm{GeV}$, as $B=4.82$.

It is tempting to conceive the masses $m_{q u}$ as the common conventional constituent masses $m_{u}$ that carry the energy of the pion. The adoption of $m_{\pi}^{\prime}$ as reference and the identification of $m_{\pi}^{\prime}$ as the (non relativistic) lab frame equivalent of the (relativistic) weak interaction boson $\hbar \omega_{W}$ requires a more careful inspection. The bond between the two quarks is somewhat different from the bond between the electron and the positron in the positronium. All we know about the quarks is that the decay from the lowest state of energy of the quark-antiquark bond results in a lab frame rest mass $m_{\pi}$ with massive energy $m_{\pi}^{\prime}=m_{\pi} c^{2}$. This decay is mediated by the weak interaction boson $\hbar \omega_{W}$. Hence, It is fair to suppose that its (relativistic) energy represents the equivalent of the nonrelativistic lab frame rest mass, i.e., $\hbar \omega_{W} \rightarrow m_{\pi}^{\prime}$. The energy of the weak interaction boson is composed by two components. These are the binding energy between the quarks and the equivalent constituent energies from the two quarks. Considering that the ground state energy of the (an)harmonic oscillator that they build amounts to $\hbar \omega_{W} / 2$, the other half has to be attributed to the constituing masses. Because the two masses constitute a one-body equivalent of a two-body oscillator, and considering that the effective mass $m$ of the single body is made up as, $m=m_{1} m_{2} /\left(m_{1}+m_{2}\right)$, the constituent rest mass energies $m_{q u}^{\prime}$ of the quarks in the pion equivalent of the positron can be established from,

$$
\frac{\hbar \omega_{W}}{2}=\frac{m_{\pi}^{\prime}}{2}=\frac{m_{q u}^{\prime 2}}{2 m_{q u}^{\prime}} \rightarrow m_{q u}^{\prime}=m_{\pi}^{\prime} .
$$

Hence, applying (A-12) to (A-11),

$$
\Delta U_{\pi \rho}=B m_{\pi}^{\prime} ; B=4.82 \text {. }
$$

In terms of the common conventional definition of the constituent mass $\left(m_{u} \neq m_{q u}\right)$ as used by Griffiths for mesons [7],

$$
\begin{aligned}
& m_{\pi}^{\prime}=2 m_{u}^{\prime}-3 A m_{u}^{\prime} \rightarrow m_{\pi}^{\prime}=(2-3 A) m_{u}^{\prime} \\
& m_{\rho}^{\prime}=2 m_{u}^{\prime}+A m_{u}^{\prime}
\end{aligned}
$$

Application of (A-14) on (A-13) yields a relationship between $B$ and $A$ as 


$$
\Delta U_{\pi \rho}=4 A m_{u}^{\prime}=B m_{\pi}^{\prime}=B(2-3 A) m_{u}^{\prime} \rightarrow 4 A=B(2-3 A) .
$$

Taking the rest mass energy of the pion $m_{\pi}^{\prime}=140 \mathrm{MeV}$ as a reference, the constituent masses $m_{u}$ of the $u / d$ quarks are calculated from (A-13)- (A-15) as $A=0.52$ and $m_{u}^{\prime}=314 \mathrm{MeV}$. These values are close to the empirical values $A \approx 0.51, m_{u}^{\prime}=310 \mathrm{MeV}$ and, mentioned in Griffith's textbook as a result of numerical fitting.

Quite interestingly, this result can be interpreted and confirmed in a curious way. It will be clear that the decay from the vector state of the archetype meson into the pseudoscalar state is a boson process next to the boson process mediated by the $W$ bosons in the bond between the quark and the antiquark in the pion state. It is therefore fair to state that the boson energy represented by $A m_{u}^{\prime}$ corresponds with the energy of the $Z$ boson in virtual state. Hence, denoting $m_{Z}^{\prime \prime}$ as the virtual state of the $Z$ boson, we have

$$
A m_{u}^{\prime}=m_{Z}^{\prime \prime}
$$

Considering that $m_{W}^{\prime}$ is the relativistic state of $m_{\pi}^{\prime}$, we may calculate $m_{Z}^{\prime \prime}$ from $m_{Z}^{\prime}$ from

$$
m_{Z}^{\prime \prime}=m_{Z}^{\prime}\left(\frac{m_{\pi}^{\prime}}{m_{W}^{\prime}}\right)
$$

Hence from (A-14), (A-16) and (A-17),

$$
m_{u}^{\prime}=\frac{1}{2} m_{\pi}^{\prime}\left(1+3 \frac{m_{Z}^{\prime}}{m_{W}^{\prime}}\right) ; \quad A=\frac{2}{3+m_{W}^{\prime} / m_{Z}^{\prime}}
$$

It has to be emphasized here that solving (A-13) would have been the more principal way of calculating these values. Adopting the view that this would result in the identification of the $Z$ boson has just been an easy escape from the problem to solve (A-13) in a straightforward cumbersome analytical way. It demonstrates, though, that the $Z$ boson is not a true elementary particle.

Taking the rest mass energy of the pion $m_{\pi}^{\prime}=140 \mathrm{MeV}$ as a reference, the constituent masses $m_{u}$ of the $u / d$ quarks are calculated from (A-18), under consideration of $m_{W}^{\prime}=80.4 \mathrm{GeV}$ and $m_{Z}^{\prime}=91.2$ $\mathrm{GeV}$ as $A=0.515$ and $m_{u}^{\prime}=308 \mathrm{MeV}$. These values are even closer to the empirical values $A \approx$ $0.51, m_{u}^{\prime}=310 \mathrm{MeV}$ mentioned in Griffith's textbook as a result of numerical fitting. We may go a step further by invoking the mass relationships due to the nuclear spin interactions as derived in Griffith's textbook in conjunction with the theoretically established ratio 3.57 of the kaon mass over the pion mass, listed in Table I. The constituent mass value for the s quark is calculated from,

$$
m_{\kappa}^{\prime}=m_{u}^{\prime}+m_{S}^{\prime}-3 \frac{m_{u}^{\prime 2}}{m_{u}^{\prime} m_{s}^{\prime}} A m_{u}^{\prime}=3.57 m_{\pi}^{\prime} \rightarrow m_{s}^{\prime}=489 \mathrm{MeV}
$$


The calculated values of the charmed quark $(c)$ and the bottom quark $(b)$ can be found paragraph 6. As noted before, the top quark $(t)$ is out of scale. As has been discussed in paragraph 7 , this is due to its different origin.

This means that the constituent masses of the quarks can be theoretically derived from a single reference for which we have adopted the rest mass of the archetype meson. This rest mass is the lab frame value of the weak boson interaction boson that binds the quark in the archetype meson. This implies that there is no reason to consider the quark flavors as elementary. Note the difference with the calculation in Griffiths' textbook, which is purely empirically based and denoted as "shaky", but nevertheless rather accurate. The quark model based upon the polarisable dipole moment of Dirac's third particle, has given it now a clear theoretical basis.

\section{References}

[1] M. Gell-Mann and Y. Ne-eman, The Eightfold Way: A Review, A.A. Benjamin Inc. New-YorkAmsterdam , 168 (1964)

[2] Gell-Mann-Okubo mass formula, https://en.wikipedia.org/wiki/Gell-Mann/Okubo_mass_formula

[3] A.D. Sakharov, Sov. Phys. JETP 51 (6), (1980)

[4] M.A. Shifman, A.I.Vainshtein and V.I. Zakharov, Nucl. Phys. B. 147,385, 448 (1979)

[5] S.R. Sharpe, pdg.libl.gov/2015/reviews/rpp2015-rev-lattice-qcd.pdf (2016)

[6] E. Roza, Found. Phys. 50, 829 (2020)

[7] D.J. Griffiths, Introduction to Elementary Particles, Wiley-VCH, Weinheim Germany (2004)

[8] P.A.M. Dirac, Proc. Royal Soc. London, A 117 (1928)

[9] E. Roza, Results in Physics, 6, 149 (2016)

[10] P.W. Higgs, Phys. Rev. Lett. 13, 508 (1964)

[11] E. Roza, Phys. Essays 25, 191, (2012)

[12] S. Wolfram, Mathematica (Addison-Wesley Publishing Inc. Redwood City, Ca (1991)

[13] T. Nakano, N. Nishijima, Progress of Theor. Physics 10 (5), 581 (1953)

[14] M. Gell-Mann, II Nuovo Cimento 4 (S2), 848 (1956)

[15] ] D.J. Griffiths, Introduction to Quantum Mechanics, Prentice-Hall Inc. , New Jersey (1995)

[16] M. Karliner and H.J. Lipkin, ArXiv:hep-ph/0307243v2

[17]] E. Roza, Phys. Essays, 26, 138 (2013); Phys. Essays, 27, 340 (2014)

[18] E. Comay, $H^{0}$ (Higgs) and other heavy particles, http://www.tau.ac.il/ elicomay/Unbiased.html

[19] E. Roza, Phys.Essays,24, 395 (2011)

[20] P. Debye and E. Hückel, Physik Zeitschrift, vol. 24, 9, 185 (1923)

[21] E. Roza, Phys. Essays, 26, 138 (2013)

[22] E. Roza, www.preprints.org, doi:10.20944/preprints202006.0304.v2 (2021)

[23] D.J. Griffiths, Am. J. Phys. 50, 698 (1982)

[24] C. Cohen-Tannoudji, B. Diu, Quantum Mechanics, Hermann, Paris (1977)

[25] A. Czarnecki, K. Melnikov, A. Yelkovsky, Phys. Rev. Letters, 82, 311 (1999)

[26] Roza, E, https://www.preprints.org/manuscript/202002.0275/v3 